\title{
A Cognitive Radio Spectrum Sensing Method for an OFDM Signal Based on Deep Learning and Cycle Spectrum
}

\author{
Guangliang Pan $\mathbb{D}^{1}{ }^{1}$ Jun Li $\mathbb{D}{ }^{2}$ and Fei Lin $^{2}$ \\ ${ }^{1}$ School of Electrical Engineering and Automation, Qilu University of Technology (Shandong Academy of Sciences), \\ Jinan 250353, China \\ ${ }^{2}$ School of Electronic and Information Engineering (Department of Physics), Qilu University of Technology (Shandong Academy \\ of Sciences), Jinan 250353, China
}

Correspondence should be addressed to Jun Li; rogerjunli@sdu.edu.cn

Received 13 September 2019; Accepted 14 February 2020; Published 6 March 2020

Academic Editor: Jintao Wang

Copyright (C) 2020 Guangliang Pan et al. This is an open access article distributed under the Creative Commons Attribution License, which permits unrestricted use, distribution, and reproduction in any medium, provided the original work is properly cited.

In a cognitive radio network (CRN), spectrum sensing is an important prerequisite for improving the utilization of spectrum resources. In this paper, we propose a novel spectrum sensing method based on deep learning and cycle spectrum, which applies the advantage of the convolutional neural network (CNN) in an image to the spectrum sensing of an orthogonal frequency division multiplex (OFDM) signal. Firstly, we analyze the cyclic autocorrelation of an OFDM signal and the cyclic spectrum obtained by the time domain smoothing fast Fourier transformation (FFT) accumulation algorithm (FAM), and the cyclic spectrum is normalized to gray scale processing to form a cyclic autocorrelation gray scale image. Then, we learn the deep features of layer-by-layer extraction by the improved CNN classic LeNet-5 model. Finally, we input the test set to verify the trained CNN model. Simulation experiments show that this method can complete the spectrum sensing task by taking advantage of the cycle spectrum, which has better spectrum sensing performance for OFDM signals under a low signal-noise ratio (SNR) than traditional methods.

\section{Introduction}

The emergence of the fifth-generation mobile communication network $(5 \mathrm{G})[1]$ has greatly promoted the development of broadband wireless communication [2], and orthogonal frequency division multiplex (OFDM) is one of the popular physical transmission technologies for wireless communication [3, 4]. Many of its characteristics meet the requirements of cognitive radio (CR) [5-7]. Therefore, OFDM is the preferred technology for CR communication. As the core technology of the cognitive radio network (CRN), spectrum sensing can improve spectrum utilization and alleviate spectrum resources [8-10]. Traditional spectrum sensing is mainly done by mathematical methods of signal processing, including energy detection [11], matched filter detection [12], and cyclostationary feature detection [13]. [14] proposed a spectrum sensing method based on correlation detection, the correlation of cyclic prefix (CP) was used in OFDM, and the sampled data was subjected to correlation operation. In [15], the signal and noise were estimated simultaneously by the time domain correlation function, and the estimated threshold was continuously adjusted by the estimated value to complete the spectrum sensing of an OFDM signal. In [16], the received autocorrelation function was estimated at each OFDM symbol of its symbol period, and then, the multivariate statistical theory was used to calculate the judgment amount and the decision threshold. Finally, the judgment amount and the decision threshold were compared to obtain the judgment result. In the face of complex wireless network communication environments, these methods cannot meet the needs of spectrum sensing. In [17], focusing on classifying different OFDM signals, authors proposed a two-step detection and identification method. However, this method is not combined with 
the deep learning model, and the spectrum sensing performance is general.

The contributions of this paper are summarized as follows:

(i) We analyze the cyclic autocorrelation of an OFDM signal, using the time domain smoothing fast Fourier transformation (FFT) accumulation algorithm (FAM) to achieve cyclic spectrum and transforming the spectrum sensing problem into an image processing and recognition problem

(ii) We convert the cyclic spectrum into a gray scale image, transforming the spectral perception problem into an image processing problem

(iii) We adopt an improved CNN based on LeNet-5 to generate a spectrum sensing model to complete spectrum sensing

The remainder of the paper is organized as follows. Section 2 introduces the related work. Section 3 analyzes the cyclic autocorrelation of an OFDM signal, and cyclic spectrum is obtained by the FAM algorithm, which is converted to a gray scale image. Section 4 presents the OFDM spectrum sensing model designs based on the improved CNN classical structure LeNet-5. Section 5 carries out simulation experiments and performance evaluation on the proposed new method. In the end, we conclude this article in Section 6.

\section{Related Work}

The existing spectrum sensing methods mainly use the neural network as a classifier, and the data set is a complex feature statistic, and the sensing effect is not ideal [18-21]. [22] proposed multigene genetic programming- (MGGP-) based feature engineering was conducted to transform the cumulants of the received signals into highly discriminative features, and then, authors use a logistic regression classifier to achieve classification of overlapping signal modulation. [23] presented a study about the possibility of implementing approximations to the Neyman-Pearson (NP) detector with C-Support Vector Machines (C-SVM) and 2C-SVM. It was based on obtaining the functions that these learning machines approximate to after training to minimize the empirical risk, and on the possible implementation of the NP detector with these approximated functions. In [24], authors tested the application of deep neural networks to the automatic modulation classification in AWGN and flatfading channel. Three training inputs were used: (1) inphase and quadrature (I-Q) constellation points, (2) the centroids of constellation points employing the fuzzy C-means algorithm to I-Q diagrams, and (3) the high-order cumulants of received samples. The unsupervised learning from these data sets was done using the sparse autoencoders, and a supervised Soft-max classifier was employed for the classification. The above intelligent algorithms are limited to long training time and only propose applications in signal recog- nition and classification, which are not applicable in spectrum sensing.

\section{Spectrum Sensing and Cyclic Spectrum}

3.1. OFDM Cyclic Autocorrelation and FAM Algorithm. Targeting the OFDM signal model, the equivalent baseband signal can be expressed as

$$
s(t)=\sum_{i=0}^{N-1} d_{i} \exp \left(j 2 \pi \frac{i}{T} t\right)
$$

where $t \in[0, T]$.

After OFDM signals pass through AWGN channel, the complex baseband signal is obtained as

$$
r(t)=s(t)+h(t)
$$

where $h(t)$ represents zero mean white noise.

The OFDM signal model is obtained as

$$
x(t)=\sum_{k} \sum_{l=0}^{G-1} c_{k, l} p\left(t-l T_{\mathrm{c}}-k T_{\mathrm{s}}\right),
$$

where $G$ represents the total length of OFDM signal, $T_{c}$ represents the chip time, $T_{s}$ represents the effective period, $p(t)$ represents the rectangular pulse, $c_{k, l}$ represents the $l$ th sampling point of the $k$ th OFDM symbol inserted into $\mathrm{CP}$.

The time domain expression can be followed as

$$
c_{k, l}=\frac{1}{\sqrt{N}} \sum_{n=0}^{N-1} a_{k, n} \exp \left[j 2 \pi(l-D) \frac{n}{N}\right],
$$

where $N$ represents the effective length and $D$ represents the length of inserted $l=0,1, \cdots, G-1 . a_{k, n}$ represents the modulated data on the $n$th subcarrier of the $k$ th OFDM signal in the frequency domain, $n=0,1, \cdots, N-1$.

In the $k$ th OFDM symbol of the signal, the autocorrelation of the time domain data $c_{k, l}$ with delay sample point $n_{\tau}$ $\left(0 \leq n_{\tau} \leq N\right)$ can be followed as

$$
\begin{aligned}
E\left(c_{k, l}, c_{k, l-n_{\tau}}^{*}\right)= & \frac{1}{N} \sum_{n=0}^{N-1} E\left(a_{k, n}, a_{k, n}^{*}\right) \exp \left[j 2 \pi(l-D) \frac{n}{N}\right. \\
& \left.-j 2 \pi\left(l-n_{\tau}-D\right) \frac{n}{N}\right]=\frac{\sigma_{a}^{2}}{N} \sum_{n=0}^{N-1} \exp \left[j 2 \pi n_{\tau} \frac{n}{N}\right] \\
= & \begin{cases}\sigma_{a}^{2}, & n_{\tau}=0, N, \\
0, & n_{\tau}=\text { others. }\end{cases}
\end{aligned}
$$

Regardless of other interference factors, the correlation function from equation (3) is 


$$
\begin{aligned}
R_{x}(t, \tau)= & E\left[x(t) x^{*}(t-\tau)\right]=\sum_{k} E\left[\sum _ { l = 0 } ^ { G - 1 } c _ { k , l } p \left(t-l T_{\mathrm{c}}\right.\right. \\
& \left.\left.-k T_{\mathrm{s}}\right) \sum_{l=0}^{G-1} c_{k, l-n_{\tau}}^{*} p^{*}\left(t-l T_{\mathrm{c}}-k T_{\mathrm{s}}-\tau\right)\right],
\end{aligned}
$$

where $\tau=n_{\tau} T_{c}$.

Inserting the $\mathrm{CP}$ introduces the correlation characteristic. It can be known from equation (6) that the OFDM signal has two cycle periods: the small period is $T_{\mathrm{c}}$ and the large period is $T_{\mathrm{s}}$, and the expression can be followed as

$$
R_{x}(t, \tau)= \begin{cases}\sigma_{a}{ }^{2} \sum_{k} \sum_{l=0}^{G-1} p\left(t-l T_{\mathrm{c}}-k T_{\mathrm{s}}\right) p^{*}\left(t-l T_{\mathrm{c}}-k T_{\mathrm{s}}-\tau\right), & |\tau|<T_{\mathrm{c}}, \\ \sigma_{a}{ }^{2} \sum_{k} \sum_{l=N}^{G-1} p\left(t-l T_{\mathrm{c}}-k T_{\mathrm{s}}\right) p^{*}\left(t-l T_{\mathrm{c}}-k T_{\mathrm{s}}-\tau_{N}\right), & \left|\tau_{N}\right|<T_{\mathrm{c}} \\ 0, & \tau=\text { others }\end{cases}
$$

where $\tau_{N}=|\tau|-N T_{c}$.

It is proved from the above that the OFDM signal has cyclic characteristics, $R_{x}(t, \tau)$ has periodicity, and further
$R_{x}(t, \tau)$ pairs do discrete Fourier transform (DFT), the expression can be expressed as

$$
\left|R_{x}^{\alpha}(\tau)\right|= \begin{cases}\frac{\sigma_{\mathrm{a}}^{2}}{T_{\mathrm{c}}}\left|\frac{\sin \left[\pi \alpha\left(T_{\mathrm{c}}-|\tau|\right)\right]}{\pi \alpha}\right|, & \alpha=\frac{m}{T_{\mathrm{c}}},|\tau| \leq T_{\mathrm{c}}, m \in Z, \\ \frac{\sigma_{\mathrm{a}}^{2}}{T_{\mathrm{s}}}\left|\frac{\sin \left(\pi \alpha T_{\mathrm{c}} D\right)}{\sin \left(\pi \alpha T_{\mathrm{c}}\right)}\right|\left|\frac{\sin \left[\pi \alpha\left(T_{\mathrm{c}}-\left|\tau_{N}\right|\right)\right]}{\pi \alpha}\right|, & \alpha=\frac{m}{T_{\mathrm{s}}},\left|\tau_{N}\right| \leq T_{\mathrm{c}}, m \in Z, \\ 0, & \tau=\text { others, }\end{cases}
$$

where $\alpha$ represents the cycle frequency.

It can be seen from the above that the OFDM signal has second-order cyclostationary characteristics. The OFDM signal has two cycles of $T_{\mathrm{c}}$ and $T_{\mathrm{s}}$, and $R_{x}(t, \tau)$ has periodicity. According to the nature of Fourier transform, the Fourier transform of $t$ will appear discrete spectrum line at $\alpha=m /$ $T_{\mathrm{c}}$ and $\alpha=m / T_{\mathrm{s}}$.

We implement the cyclic spectrum by FAM algorithm. The flow of the implementation steps of algorithm can be shown in Figure 1.

Through the above cyclic autocorrelation analysis, the FAM algorithm is used to simulate in simulation software, and simulation parameters are shown in Table 1.

The three-dimensional cyclic spectrum of OFDM under $H_{0}\left(H_{0}\right.$ : it represents the absence of OFDM signal; that is, the authorized user does not exist.) and $H_{1}\left(H_{1}\right.$ : it represents the presence of OFDM signal, that is, the presence of authorized users.) are obtained, as shown in Figures 2 and 3.

3.2. Spectrum Sensing Model. The CR user discovers and utilizes opportunistically the available spectrum opportunities to access dynamically the idle licensed spectrum. According to the available spectrum opportunities of CR users, the CR spectrum perception can be defined as a binary hypothesis model as follows:

$$
y(t)= \begin{cases}n(t), & H_{0}, \\ g s(t)+n(t), & H_{1} .\end{cases}
$$

The binary hypothesis model has false alarm errors $P_{\mathrm{f}}$ and missed detection errors $P_{\mathrm{m}}$, which are represented by false alarm probability and missed detection probability as follows:

$$
\left\{\begin{array}{l}
P_{\mathrm{f}}=\operatorname{Pr}\left(Y>\lambda \mid H_{0}\right), \\
P_{\mathrm{m}}=\operatorname{Pr}\left(Y<\lambda \mid H_{1}\right),
\end{array}\right.
$$

where $Y$ represents the decision statistic and $\lambda$ represents the threshold. According to the NP criterion, in order to improve the spectrum sensing performance of CR users, it is necessary to reduce $P_{\mathrm{m}}$ as much as possible under certain $P_{\mathrm{f}}$ constraints.

The three-dimensional cyclic spectrum is normalized and gradated, and a two-dimensional cyclic autocorrelation gray 


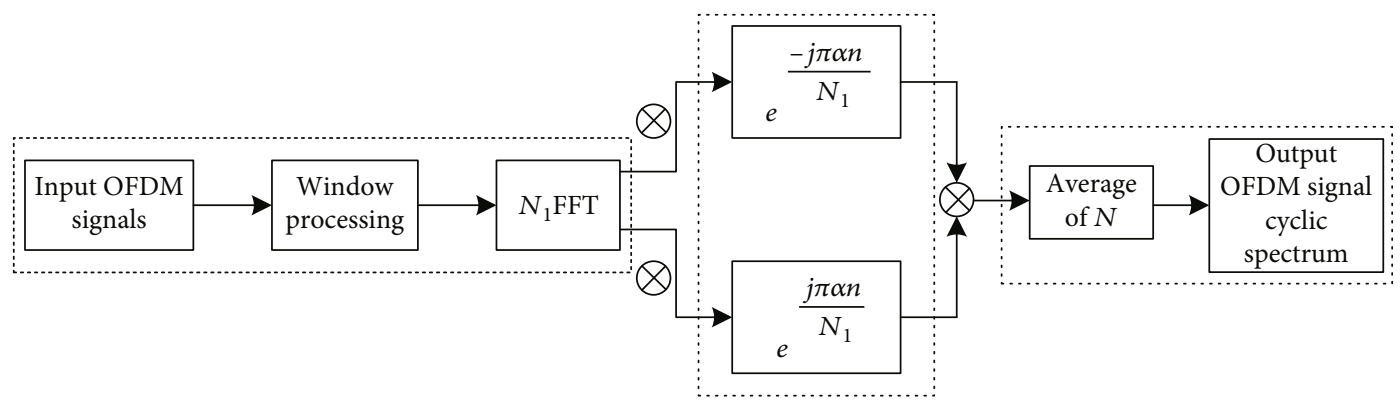

Figure 1: Process.

TABLE 1: Simulation experiment parameters.

\begin{tabular}{lc}
\hline Parameters & Data \\
\hline Number of OFDM in one frame & 20 \\
Number of subcarriers $(N)$ & 128 \\
Proportion of cyclic prefix & $1 / 4$ \\
Symbol rate & $12.5 \mathrm{kbps}$ \\
Carrier frequency & $1 \times 10^{6} \mathrm{~Hz}$ \\
Sampling frequency & $4 \times 10^{7} \mathrm{~Hz}$ \\
Chip rate & $5 \times 10^{5} \mathrm{~Hz}$ \\
Smooth points & 20 \\
Cyclic frequency resolution & $2 \times 10^{6} \mathrm{~Hz}$ \\
\hline
\end{tabular}

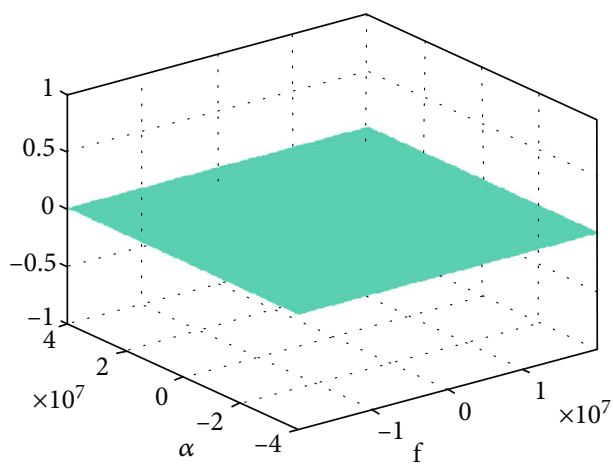

Figure 2: Cycle spectrum $H_{0}$.

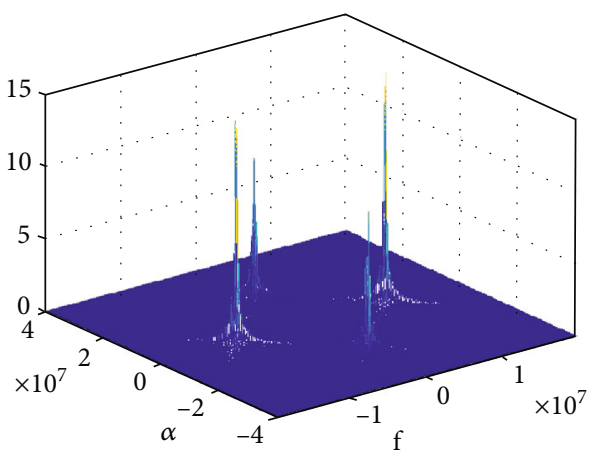

Figure 3: Cycle spectrum $H_{1}$. map containing Gaussian white noise under $H_{0}$ and $H_{1}$ are obtained, as shown in Figures 4 and 5.

The gray value is used to represent the size of the autocorrelation value (the gray value is positively correlated with the autocorrelation value). According to the distribution of twodimensional gray map under $H_{0}$ and $H_{1}$, it has four peaks under $H_{1}$, which can map the spectrum perception to image processing [25].

\section{Method}

4.1. CNN Design. The spectrum sensing of OFDM signal is completed by the deep learning framework, and the implementation framework is shown in Figure 6, which is divided into a model training process and a model testing process.

$\mathrm{CNN}$ is a model of deep learning. It has the characteristics of local connection and parameter sharing. It has strong advantages in image processing. LeNet-5 model is a classic model of CNN. We change the input of the LeNet-5 model to gray scale image of $36 * 36$ and change the size of the convolution kernel and sampling window. Spectrum sensing depends on the spectrum occupancy of authorized users, changes the output to 2 neurons, and converts to binary classification; label 0 represents $H_{0}$, and label 1 represents $H_{1}$. An improved LeNet-5 model for spectrum sensing is shown in Figure 7.

4.2. Train and Test. The CNN based on the improved LeNet5 model is trained by the error back propagation (BP) algorithm. The training process of $\mathrm{BP}$ algorithm is divided into forward calculation of data, back propagation of error, and update of weight. $\delta_{k}$ represents the partial derivative of input layer error for this layer, which is called sensitivity [26].

For forward calculation of data, the hidden layer output values are defined as

$$
\left\{\begin{array}{l}
a_{h}^{H}=W_{h}^{H} \times X_{i}, \\
b_{h}^{H}=f\left(a_{h}^{H}\right),
\end{array}\right.
$$

where $W_{h}^{H}$ represents the weight of layer $H, X_{i}$ represents the current node input, and $f(\cdot)$ represents the current layer activation function.

The output layer output values are defined as

$$
a_{k}=\sum W_{h k} \times b_{h}^{H} .
$$




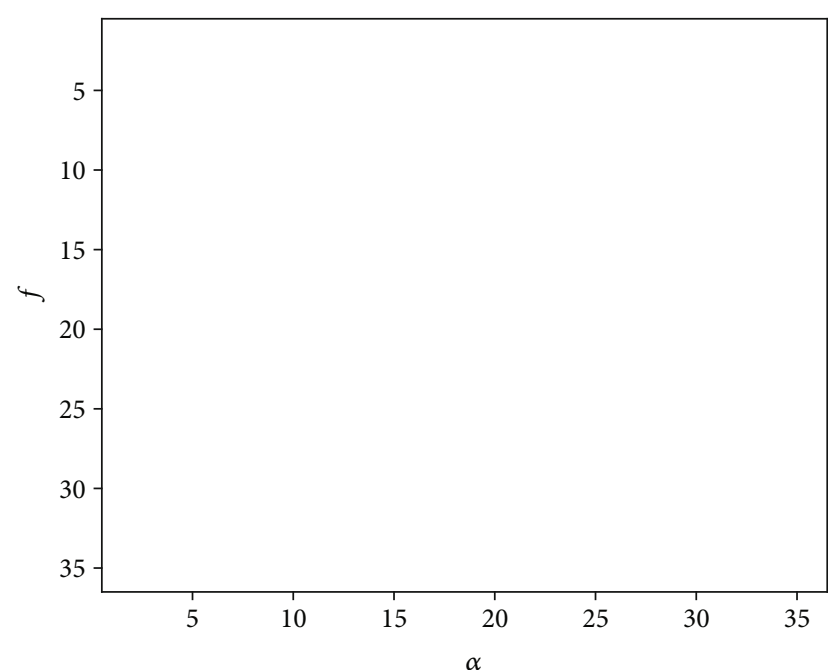

FIgURE 4: Gray scale image $H_{0}$.

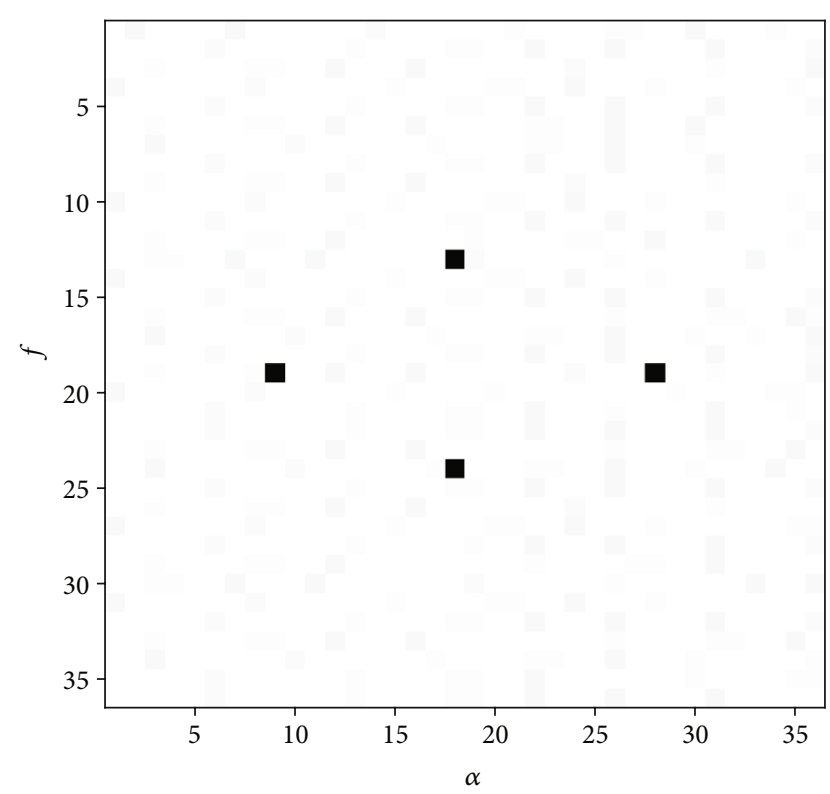

FIGURE 5: Gray scale image $H_{1}$.

In Tensorflow, the one-hot method is usually used for error back propagation and weight update. The cross entropy function is defined as

$$
\operatorname{loss}_{\mathrm{c}}=-y \log (f(x)) \text {. }
$$

The output layer feeds back to the reverse derivation of the fully connected layer. According to the one-hot method, only one value is 1 and the rest is 0 . The cross entropy can be followed as

$$
\begin{aligned}
\operatorname{loss}(f(x), y)= & -\sum y \log (f(x))=-\left(0 \times \log \left(f\left(x_{1}\right)\right)+\cdots+1\right. \\
& \left.\times \log \left(f\left(x_{n}\right)\right)\right)=-\log \left(f\left(x_{n}\right)\right) .
\end{aligned}
$$

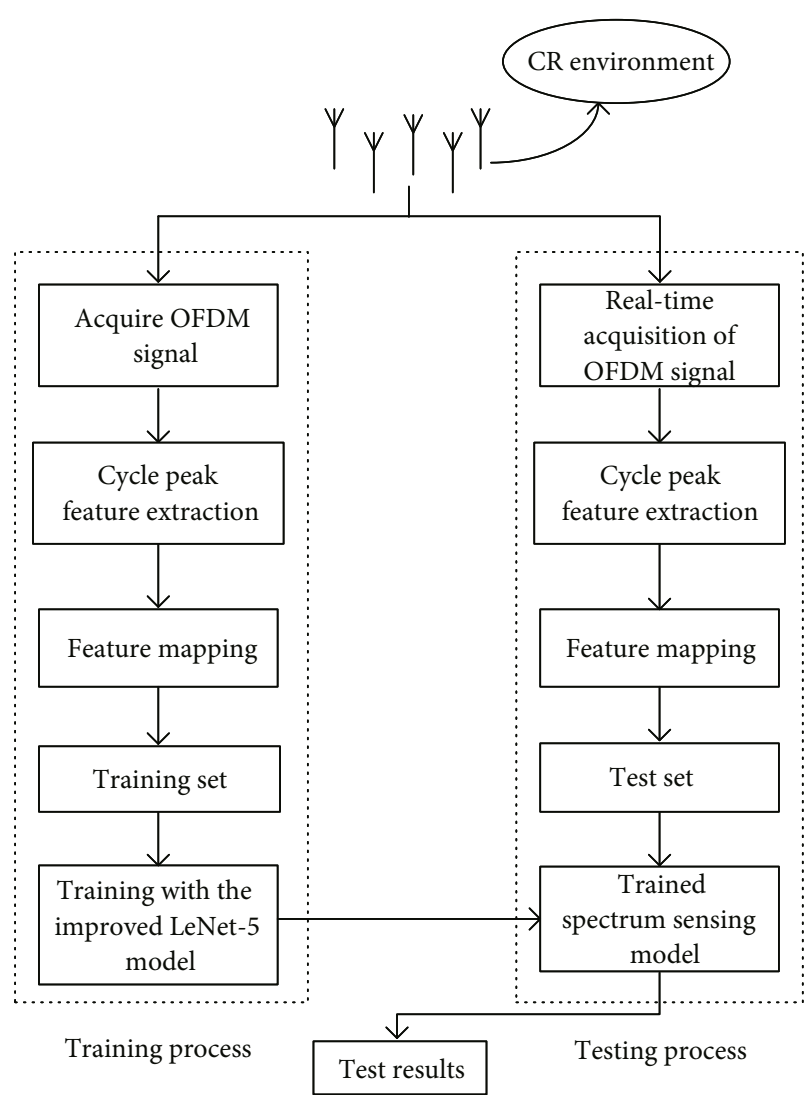

FIGURE 6: The framework of spectrum sensing.

Loss value is as follows:

$$
\text { loss }=-(y-\log (f(x))) \text {. }
$$

Let $y=1$ get

$$
\text { loss }=-(1-\log (f(x))) \text {. }
$$

The output layer uses Soft-max, and the formula for the full connection layer weight update is as follows:

$$
\frac{\delta \text { loss }}{\delta W}=-\frac{1}{m} \times(1-f(x)) f^{\prime}(x)+\lambda W
$$

The pooled layer feeds back to the reverse derivation of the convolutional layer, and its convolutional layer sensitivity can be followed as

$$
\delta_{j}^{l}=\operatorname{pool}\left(\delta_{j}^{l+1} * h^{\prime}\left(a_{j}^{l}\right)\right)
$$

where $*$ represents the dot multiplication. The convolutional layer feeds back to the reverse derivation of pooling layer, assuming that $l$ is a pooling layer, $l+1$ is a convolutional 


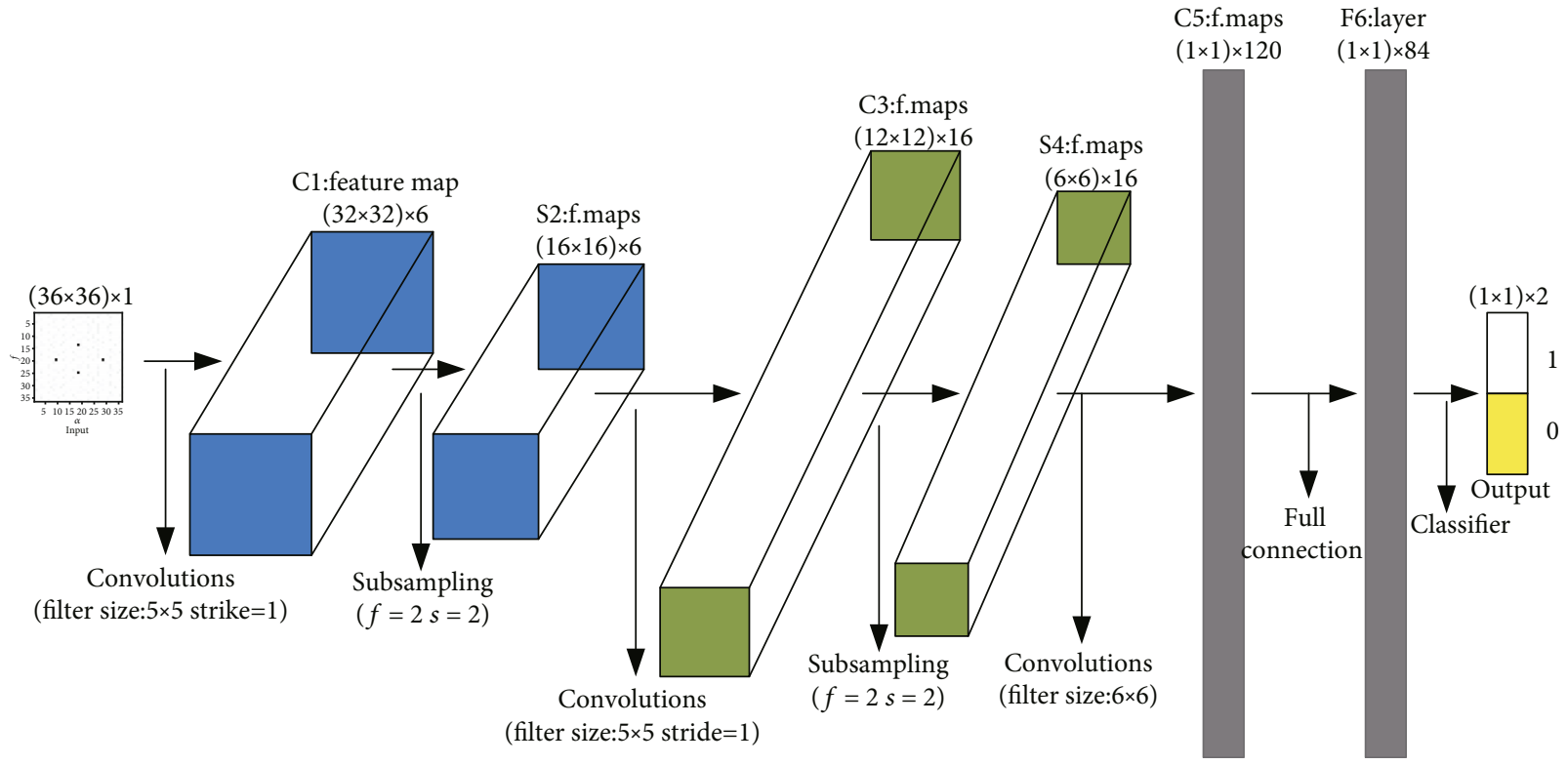

FIGURE 7: Improved structural model of CNN.

layer, and the convolutional layer has $m$ features, and the sum of pooling layer sensitivity can be followed as

$$
\delta_{j}^{l}=\sum_{j}^{m} \delta_{j}^{l+1} \odot K_{i j},
$$

where $\odot$ represents a convolution operation. The sensitivity is obtained by the above calculation, and then, weights and offsets in the CNN can be followed as

$$
\begin{aligned}
& \frac{\delta \text { loss }}{\delta W_{i j}}=X_{i} \delta_{j}^{i+1}, \\
& \frac{\delta \text { loss }}{\delta b_{i j}}=\Sigma \delta_{j}^{i+1} .
\end{aligned}
$$

After BP algorithm trains CNN, it is verified by the test set.

We take $N$ pairs of test sets $\left\{\left(x_{1}, y_{1}\right),\left(x_{2}, y_{2}\right), \cdots,\left(x_{i}, y_{i}\right)\right\}$, and the difference between the predicted value and the true value can be followed as

$$
\Omega=\left\|Y_{W, b}\left(x_{i}\right)-y_{i}\right\|
$$

where $Y_{W, b}\left(x_{i}\right)$ represents the output value of last layer of $\mathrm{CNN}$ model, $W$ represents the weight, $b$ represents the offset.

Then, the accuracy of this test set is obtained as

$$
\text { Accuracy }=\frac{n}{N},
$$

where $n$ represents the number of tests meeting the $\Omega<\Omega_{\text {th }}$ condition.

It can be seen from equation (22) that the higher accuracy, the better performance of spectrum sensing.
TABle 2: Data set.

\begin{tabular}{lcc}
\hline Type & Label & Effective sample \\
\hline \multirow{2}{*}{ Train set } & 0 & 2000 \\
& 1 & 2000 \\
\hline \multirow{2}{*}{ Test set } & 0 & 200 \\
& 1 & 200 \\
\hline
\end{tabular}

TABLE 3: CNN parameter setting.

\begin{tabular}{lc}
\hline Parameter & Value \\
\hline Training set & $4000(36 \times 36)$ \\
Test set & $400(36 \times 36)$ \\
Number of network layers $(N)$ & 6 \\
Activation function & Sigmoid \\
Learning efficiency $(\alpha)$ & 0.001 \\
Weight fading factor $(\lambda)$ & 0.1 \\
Test error threshold $\left(\Omega_{\mathrm{th}}\right)$ & 0.05 \\
Minibatch & 20 \\
\hline
\end{tabular}

\section{Experiment and Analysis}

This section simulates the performance evaluation of proposed spectrum sensing method to verify its feasibility. The OFDM frame is built in simulation software, and the OFDM signal is generated by the analog transmitter.

As shown in Table 2, labels 0 and 1 denote the state of channel as idle and busy, respectively. The balance of positive and negative samples is $1: 1$. The channel setting is referenced [27]. After through the Rayleigh fading channel and the Gaussian white noise channel, the data sampled by receiving end is preprocessed according to the second section. We complete the train and test of model in TensorFlow 
TABLe 4: Design CNN with 5 different network structures.

\begin{tabular}{lcccccccccc}
\hline & \multicolumn{2}{c}{ C1 } & \multicolumn{2}{c}{ S2 } & \multicolumn{2}{c}{ C3 } & \multicolumn{2}{c}{ S4 } & \multicolumn{2}{c}{ C5 } \\
Num & Con-kernel & Output & S-window & Output & Con-kernel & Output & S-window & Output & Con-kernel & Output \\
\hline 1 & $(3 \times 3) \times 6$ & $(34 \times 34) \times 6$ & $2 \times 2$ & $(17 \times 17) \times 6$ & $(3 \times 3) \times 16$ & $(15 \times 15) \times 16$ & $2 \times 2$ & $(8 \times 8) \times 16$ & $(8 \times 8) \times 120$ & $(1 \times 1) \times 120$ \\
2 & $(4 \times 4) \times 6$ & $(33 \times 33) \times 6$ & $2 \times 2$ & $(17 \times 17) \times 6$ & $(4 \times 4) \times 16$ & $(14 \times 14) \times 16$ & $2 \times 2$ & $(7 \times 7) \times 16$ & $(7 \times 7) \times 120$ & $(1 \times 1) \times 120$ \\
3 & $(5 \times 5) \times 6$ & $(32 \times 32) \times 6$ & $2 \times 2$ & $(16 \times 16) \times 6$ & $(5 \times 5) \times 16$ & $(12 \times 12) \times 16$ & $2 \times 2$ & $(6 \times 6) \times 16$ & $(6 \times 6) \times 120$ & $(1 \times 1) \times 120$ \\
4 & $(6 \times 6) \times 6$ & $(31 \times 31) \times 6$ & $2 \times 2$ & $(16 \times 16) \times 6$ & $(6 \times 6) \times 16$ & $(11 \times 11) \times 16$ & $2 \times 2$ & $(6 \times 6) \times 16$ & $(6 \times 6) \times 120$ & $(1 \times 1) \times 120$ \\
5 & $(7 \times 7) \times 6$ & $(30 \times 30) \times 6$ & $2 \times 2$ & $(15 \times 15) \times 6$ & $(7 \times 7) \times 16$ & $(9 \times 9) \times 16$ & $2 \times 2$ & $(5 \times 5) \times 16$ & $(5 \times 5) \times 120$ & $(1 \times 1) \times 120$ \\
\hline
\end{tabular}

deep learning framework. The performance of spectrum sensing is determined by two aspects. On the one hand, it is evaluated by $P_{\mathrm{f}}$ and $P_{\mathrm{m}}$ mentioned in the second section, and the probability of detection $P_{\mathrm{d}}\left(P_{\mathrm{d}}=1-P_{\mathrm{m}}\right)$ is derived from $P_{\mathrm{m}}$. On the other hand, it is evaluated by the training time and test time of $\mathrm{CNN}$, which is the efficiency of perception. The parameter settings of CNN are shown in Table 3.

5.1. Analysis of the CNN Model. Since the structure of CNN model will affect training and test results, the detection rate of spectrum sensing will change.

By modifying the size of convolution kernel, we design five different CNN structures, the input data set remains unchanged. The different layer structure can be shown in Table 4. The CNN models of five different structures are trained under the same training set and test set as in Table 3. Under the same training times, the accuracy and total loss (cross entropy loss and regularization loss) of the training process of five different structures $\mathrm{CNN}$ model are statistically analyzed. The performance curve can be shown in Figures 8 and 9.

It can be seen from Figure 8 that under the maximum pooling aggregation mode, as the number of training increases, the robustness of five different $\mathrm{CNN}$ structures are continuously enhanced, when the number of training is 10001 (more the optimization effect of high training times will be better, but this paper only takes 10001 times and has no effect on the research problem). No. 3 and no. 4 are the best relative to other three structures, and the actual training accuracy is 0.96 . Meanwhile, the total loss corresponding to No. 3 and no. 4 is the lowest as seen in Figure 9.

5.2. Performance Comparison Analysis of Algorithms. [22] uses logistic regression to distinguish signals, and [23] uses SVM to achieve radar signal recognition. Our method is compared with these two machine learning algorithms. The signal is set to Gaussian white noise signal and OFDM signal. Under different sample sizes $(90 \%$ of the data is used for training, and $10 \%$ of the data is used for testing), we adopt the same data set, and the Performance of three algorithms are shown in Table 5.

It can be seen from Table 5 that the CNN spectrum sensing method has obvious advantages in terms of various performances compared with others. The CNN is better than other two algorithms in image classification processing. The machine learning algorithm is good, and the OFDM cyclic spectrum feature is more suitable for CNN. For the test time

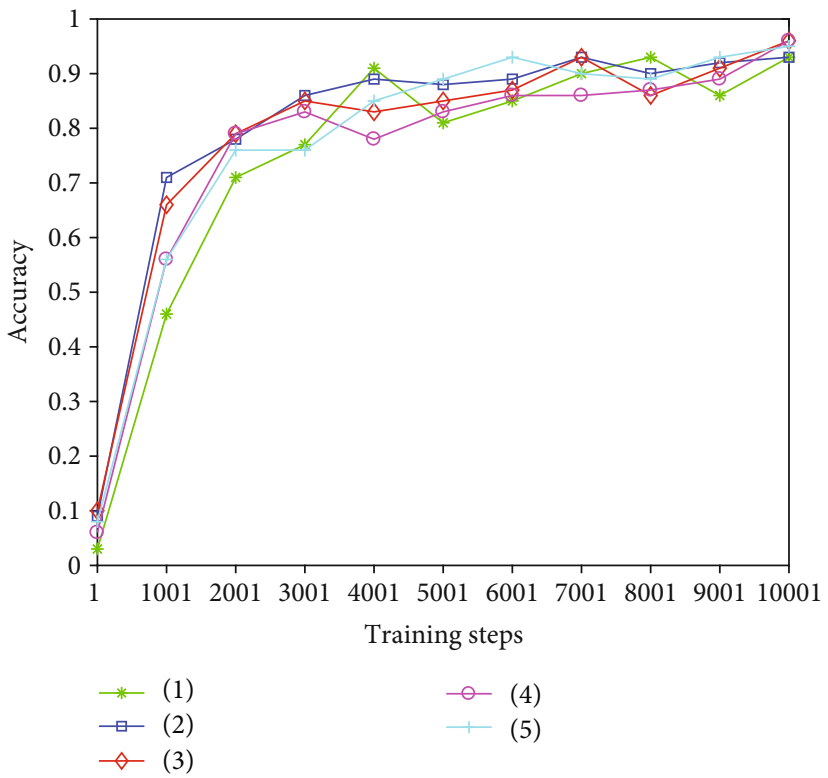

FIGURE 8: Fitting process for accuracy.

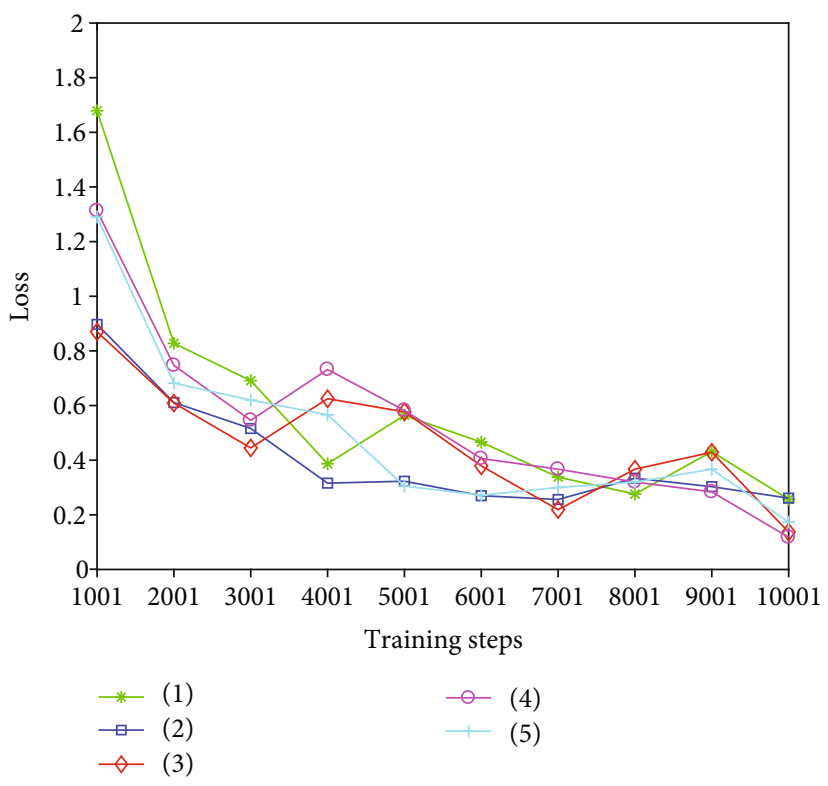

FIGURE 9: Fitting process for loss. 
TABLE 5: Comparison of three algorithms.

\begin{tabular}{|c|c|c|c|c|c|c|c|c|c|c|c|c|}
\hline \multirow[b]{2}{*}{ Data set } & \multicolumn{4}{|c|}{$\mathrm{CNN}$} & \multicolumn{4}{|c|}{ Logistic regression } & \multicolumn{4}{|c|}{ SVM } \\
\hline & $P_{\mathrm{d}}$ & $P_{\mathrm{f}}$ & $T_{\mathrm{r}}$-time $(\mathrm{s})$ & $T$-time (s) & $P_{\mathrm{d}}$ & $P_{\mathrm{f}}$ & $T_{\mathrm{r}}$-time $(\mathrm{s})$ & $T$-time (s) & $P_{\mathrm{d}}$ & $P_{\mathrm{f}}$ & $T_{\mathrm{r}}$-time $(\mathrm{s})$ & $T$-time (s) \\
\hline 1000 & 0.9478 & 0.0821 & 5.3831 & 0.3841 & 0.6387 & 0.1728 & 178.43 & 17.8 & 0.8221 & 0.0982 & 361.42 & 29.2 \\
\hline 2000 & 0.9492 & 0.0722 & 8.6242 & 0.6235 & 0.6525 & 0.1572 & 243.32 & 23.2 & 0.8292 & 0.0923 & 543.31 & 43.5 \\
\hline 3000 & 0.9539 & 0.0632 & 10.4253 & 0.8212 & 0.6693 & 0.1384 & 301.21 & 29.3 & 0.8504 & 0.0951 & 742.83 & 59.8 \\
\hline 4000 & 0.9578 & 0.0615 & 12.1235 & 0.9343 & 0.6852 & 0.1253 & 362.43 & 37.2 & 0.8525 & 0.0942 & 978.56 & 78.4 \\
\hline 5000 & 0.9632 & 0.0523 & 13.2121 & 1.0215 & 0.6971 & 0.1132 & 415.37 & 48.5 & 0.8532 & 0.0965 & 1021.35 & 87.5 \\
\hline 6000 & 0.9689 & 0.0494 & 14.5236 & 1.1245 & 0.7135 & 0.1012 & 523.41 & 56.1 & 0.8551 & 0.0937 & 1156.53 & 96.4 \\
\hline
\end{tabular}

of 100 samples is 0.3841 , the test time of a single sample is within the range allowed by spectrum sensing. Our method can meet the needs of spectrum sensing.

The training set and test set of OFDM signals are obtained through different SNR channels in the simulation platform. The logistic regression, SVM, and deep learning OFDM (DP-OFDM) spectrum sensing model are used for training and testing, and the error threshold $\Omega_{\text {th }}$ is adjusted to obtain the detection probability. As described in [28], a cyclic feature detection method based on compressed sensing, such as [11] proposed the energy detection method, and [15] proposed the autocorrelation detection method. Under the condition of false alarm probability $P_{\mathrm{f}}=0.05$, the corresponding decision thresholds are set for each of the above methods, and the above methods are simulated in the simulation platform, respectively. The performance comparison of the detection probability can be shown in Figure 10.

It can be seen from Figure 10 that under the condition of $P_{\mathrm{f}}$, we propose DP-OFDM spectrum sensing method is superior to the logistic regression and SVM algorithms. The same training set and test set are used in different SNR environments, which are less affected by noise. Below $-10 \mathrm{~dB}$, the detection probability of $\mathrm{CNN}$ is better than traditional spectrum sensing algorithms, which indicates that our method has better spectrum sensing performance under low SNR. Under the condition of high SNR, the traditional spectrum sensing algorithms have obvious performance advantages, indicating that the traditional spectrum sensing algorithms still have strong advantages under high SNR. Compressed sensing-based loop feature detection is comparable to the algorithm detection performance of this paper, but the algorithm of this paper is more advantageous. The detection rate of the traditional spectrum sensing algorithm is greatly affected by SNR, especially when SNR is below $-6 \mathrm{~dB}$.

As can be seen from Section 2, performance indicator for improving spectrum sensing is $P_{\mathrm{f}}$. Under SNR is $-12 \mathrm{~dB}$, the receiver operating characteristic (ROC) curve of $P_{\mathrm{d}}$ changing with $P_{\mathrm{f}}$ is plotted by different detection algorithms, which can be shown in Figure 11.

It can be seen from Figure 11 that the detection probability of energy detection algorithm is the lowest. The detection probability of DP-OFDM detection method is significantly higher than traditional spectrum sensing algorithms and has a stronger advantage than the two machine learning algorithms of logistic regression and SVM. The extracted

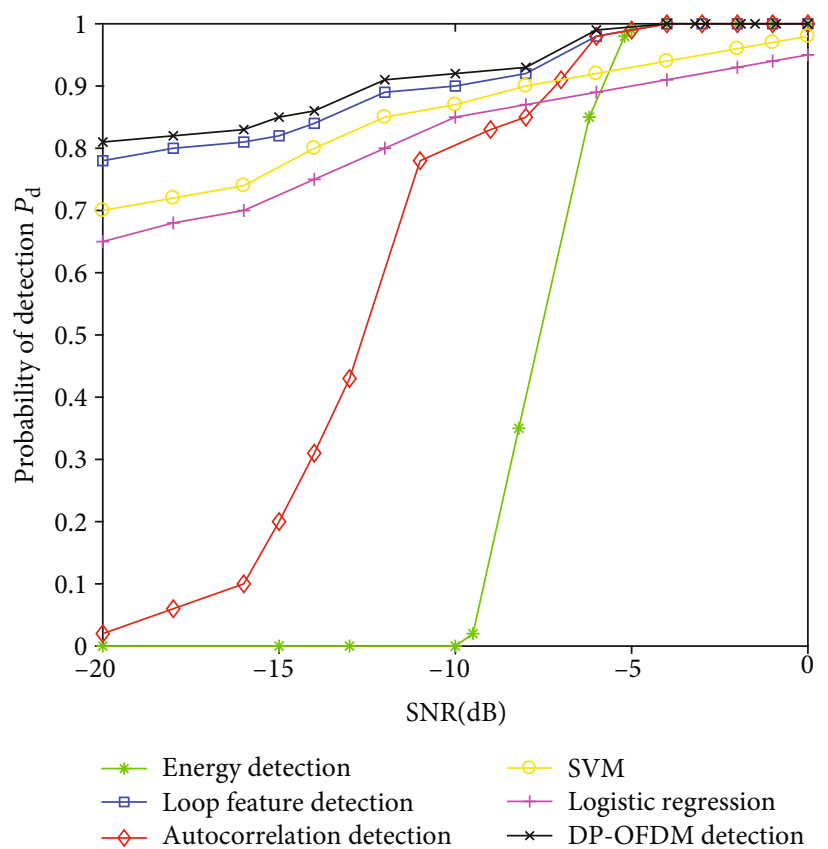

FIGURE 10: Probability of detection versus SNR for various detection algorithms $P_{\mathrm{f}}=0.05$.

autocorrelation feature of OFDM cycle can reflect the characteristics of spectrum, which is more suitable as the input of $\mathrm{CNN}$ model. It also shows that $\mathrm{CNN}$ has more powerful learning ability than other machine learning algorithms, and spectrum sensing effect is better.

\section{Conclusion}

How to effectively improve the spectrum sensing performance in complex and variable wireless environments is a challenge. In this paper, we propose a cognitive radio spectrum sensing method for OFDM signal based on deep learning and cycle spectrum. The OFDM signal cyclic spectrum feature is used as the data set of the improved CNN model, which converts the spectrum sensing problem into an image processing problem, and can better exert the powerful learning ability of CNN. Simulation results show that this method is not only better than other machine learning methods but also has higher detection probability than traditional spectrum sensing methods under low SNR. This method also 


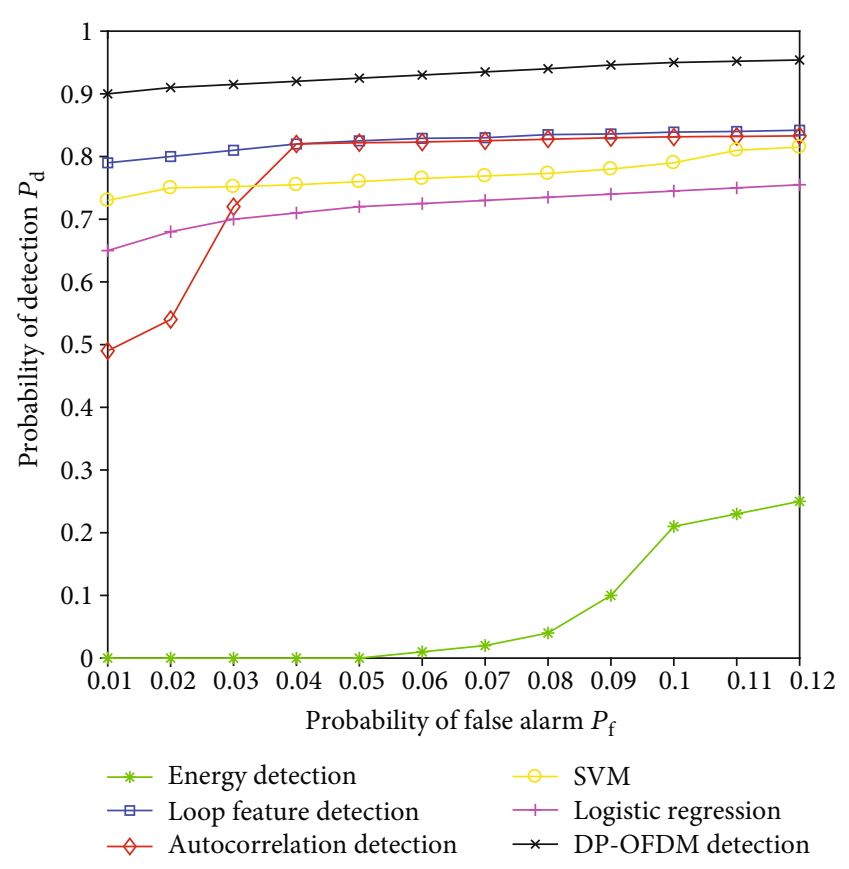

Figure 11: ROC curves of different spectral sensing methods $P_{\mathrm{d}}$ with $P_{\mathrm{f}} \mathrm{SNR}=-12 \mathrm{~dB}$.

has certain disadvantages, such as a single feature input. In short, this method has certain reference value for the application of more advanced deep learning models in the field of spectrum sensing.

\section{Nomenclature}

$h(t): \quad$ Zero mean white noise

$G$ : Total length of the OFDM signal

$T_{\mathrm{c}}$ : $\quad$ Chip time

$T_{s}: \quad$ Effective period

$p(t): \quad$ Rectangular pulse

$N: \quad$ Effective length

$D: \quad$ Length of inserted CP

$n_{\tau}$ : $\quad$ Delay sample point

$\alpha: \quad$ Cycle frequency

$P_{\mathrm{f}}: \quad$ False alarm errors

$P_{\mathrm{m}}: \quad$ Missed detection errors

$Y: \quad$ Decision statistic

$\lambda: \quad$ Threshold

$W_{h}^{H}: \quad$ Weight of layer $H$

$X_{i}$ : $\quad$ Current node input

$f(\cdot)$ : Current layer activation function

$*$ : $\quad$ Dot multiplication

$\odot$ : $\quad$ A convolution operation

$Y_{W, b}\left(x_{i}\right)$ : The output value of the last layer of the CNN model

$W: \quad$ Weight

$b$ : Offset.

\section{Data Availability}

The data used to support the findings of this study are available from the corresponding author upon request.

\section{Conflicts of Interest}

The authors declare that there are no conflicts of interest regarding the publication of this paper.

\section{Acknowledgments}

This work was supported by the Shandong Ship Control Engineering and Intelligent System Engineering Research Center 2019 Open Special Fund Project (SSCC-2019-0007).

\section{References}

[1] X. You, C. Zhang, X. Tan, S. Jin, and H. Wu, "AI for 5G: research directions and paradigms," Science China Information Sciences, vol. 62, no. 2, 2019.

[2] X. Liu, X. Zhang, M. Jia, L. Fan, W. Lu, and X. Zhai, “5G-based green broadband communication system design with simultaneous wireless information and power transfer," Physical Communication, vol. 28, pp. 130-137, 2018.

[3] H. Ye, G. Y. Li, and B. H. Juang, "Power of deep learning for channel estimation and signal detection in OFDM systems," IEEE Wireless Communications Letters, vol. 7, no. 1, pp. 114117, 2018.

[4] W. Zhang, R. Mallik, and K. Letaief, "Optimization of cooperative spectrum sensing with energy detection in cognitive radio networks," IEEE Transactions on Wireless Communications, vol. 8, no. 12, pp. 5761-5766, 2009.

[5] I. F. Akyildiz and X. Wang, "A survey on wireless mesh networks," IEEE Communications Magazine, vol. 43, no. 9, pp. S23-S30, 2005.

[6] T. Qin and C. Leung, "Fair adaptive resource allocation for multiuser OFDM cognitive radio systems," in 2007 Second International Conference on Communications and Networking in China, pp. 115-119, IEEE, Shanghai, China, 2007.

[7] H. Mahmoud, T. Yucek, and H. Arslan, "OFDM for cognitive radio: merits and challenges," IEEE Wireless Communications, vol. 16, no. 2, pp. 6-15, 2009.

[8] C. S. Karthikeyan and M. Suganthi, "Optimized spectrum sensing algorithm for cognitive radio," Wireless Personal Communications, vol. 94, no. 4, pp. 2533-2547, 2017.

[9] D. Cabric, S. M. Mishra, and R. W. Brodersen, "Implementation issues in spectrum sensing for cognitive radios," in Conference Record of the Thirty-Eighth Asilomar Conference on Signals, Systems and Computers, 2004, vol. 1, pp. 772-776, IEEE, Pacific Grove, CA, USA, USA, 2004.

[10] W.-Y. Lee and I. F. Akyildiz, "Optimal spectrum sensing framework for cognitive radio networks," IEEE Transactions on Wireless Communications, vol. 7, no. 10, pp. 3845-3857, 2008.

[11] A. Subekti, S. Sugihartono, S. Nana Rachmana, and A. B. Suksmono, "A cognitive radio spectrum sensing algorithm to improve energy detection at low SNR," Telkomnika, vol. 12, no. 3 , p. $717,2014$.

[12] D. Cabric, A. Tkachenko, and R. W. Brodersen, "Spectrum sensing measurements of pilot, energy, and collaborative detection," in MILCOM 2006-2006 IEEE Military Communications conference, pp. 1-7, IEEE, Washington, DC, USA, 2006.

[13] A. S. Kushwah and A. Shrivastava, "A review on cooperative spectrum sensing using cyclostationary feature detection for 
cognitive radio networks," International Journal of Engineering and Management Research (IJEMR), vol. 7, no. 3, pp. 474-478, 2017.

[14] K. Berbra, M. Barkat, F. Gini, M. Greco, and P. Stinco, “A fast spectrum sensing for CP-OFDM cognitive radio based on adaptive thresholding," Signal Processing, vol. 128, pp. 252261, 2016.

[15] M. Zhang, L. Wang, and L. Ding, "A fast spectrum sensing for OFDM based on adaptive thresholding," in 2017 IEEE 2nd Advanced Information Technology, Electronic and Automation Control Conference (IAEAC), pp. 2674-2678, IEEE, Chongqing, China, 2017.

[16] G. J. Mendis, J. Wei, and A. Madanayake, “Deep belief network for automated modulation classification in cognitive radio," in 2017 Cognitive Communications for Aerospace Applications Workshop (CCAA), pp. 1-5, IEEE, Cleveland, OH, USA, 2017.

[17] N. Han, G. Zheng, S. H. Sohn, and J. M. Kim, "Cyclic autocorrelation based blind OFDM detection and identification for cognitive radio," in 2008 4th International Conference on Wireless Communications, Networking and Mobile Computing, pp. 1-5, IEEE, Dalian, China, 2008.

[18] H. A. Rowley, Neural Network-Based Face Detection, Carnegiemellon Univ Pittsburgh PA Dept of Computer Science, Tech. Rep., 1999.

[19] A. L. Maas, A. Y. Hannun, and A. Y. Ng, "Rectifier nonlinearities improve neural network acoustic models," in Proc. ICML, vol. 30no. 1, p. 3, 2013.

[20] V. K. Tumuluru, P. Wang, and D. Niyato, "A neural network based spectrum prediction scheme for cognitive radio," in 2010 IEEE International Conference on Communications, pp. 1-5, IEEE, Cape Town, South Africa, 2010.

[21] A. Kumar and P. Nandha Kumar, "OFDM system with cyclostationary feature detection spectrum sensing," ICT Express, vol. 5, no. 1, pp. 21-25, 2019.

[22] Y. Jiang, S. Huang, Y. Zhang, Z. Feng, D. Zhang, and C. Wu, "Feature based modulation classification for overlapped signals," IEICE Transactions on Fundamentals of Electronics, Communications and Computer Sciences, vol. E101.A, no. 7, pp. 1123-1126, 2018.

[23] D. de la Mata-Moya, M. P. Jarabo-Amores, J. M. de Nicolás, and M. Rosa-Zurera, "Approximating the Neyman-Pearson detector with 2C-SVMs. Application to radar detection," Signal Processing, vol. 131, pp. 364-375, 2017.

[24] A. Ali, F. Yangyu, and S. Liu, "Automatic modulation classification of digital modulation signals with stacked autoencoders," Digital Signal Processing, vol. 71, pp. 108-116, 2017.

[25] B. B. Traore, B. Kamsu-Foguem, and F. Tangara, "Deep convolution neural network for image recognition," Ecological Informatics, vol. 48, pp. 257-268, 2018.

[26] Q. Yue and C. Ma, "Hyperspectral data classification based on flexible momentum deep convolution neural network," Multimedia Tools and Applications, vol. 77, no. 4, pp. 4417-4429, 2018.

[27] E. Axell, G. Leus, E. Larsson, and H. Poor, "Spectrum sensing for cognitive radio: state-of-the-art and recent advances," IEEE Signal Processing Magazine, vol. 29, no. 3, pp. 101-116, 2012.

[28] X. Liu, Q. Zhang, J. Yang, Y. Bai, and Z. Feng, "A novel algorithm to optimize sampling rate for compressed sensing," in 2013 IEEE 78th Vehicular Technology Conference (VTC Fall), pp. 1-5, IEEE, Las Vegas, NV, USA, 2013. 